\title{
A surface-to-surface scheme for 3D contact problems by boundary face method
}

\author{
Xiaomin Shu, Jianming Zhang*, Lei Han, Yunqiao Dong \\ State Key Laboratory of Advanced Design and Manufacturing for Vehicle Body, \\ College of Mechanical and Vehicle Engineering, Hunan University, Changsha, \\ 410082 China \\ * Correspondence to: Jianming Zhang \\ College of Mechanical and Vehicle Engineering, Hunan University, \\ Changsha 410082, China \\ Telephone: +86731-88823061 \\ E-mail: zhangjianm@gmail.com
}

\begin{abstract}
The surface-to-surface contact algorithm has been proposed to overcome the drawbacks of node-to-surface algorithm in finite element method implementations. In the surface-to-surface algorithm, contact constraints are imposed between sub-elements rather than between nodes, and an auxiliary plane for each element is introduced to perform overlapping area detection. This work presents a combination of the surface-to-surface algorithm and the boundary face method (BFM) for solving contact problems in three dimensions. The BFM is based on boundary integral equation and is a truly isogeometric method, as it makes direct use of the geometric information of the bounding surface of a body. Apparently, the BFM is more suitable for solving contact problems. In our implementation, the auxiliary plane is no more necessary, but replaced by the boundary faces themselves which already exist in the BFM data structure. Our implementation is natural and can more precisely match the contact conditions between faces, and therefore, higher level of accuracy can be expected. Numerical examples presented have demonstrated the advantages of the combined method.
\end{abstract}

Keywords: contact problems; surface-to-surface; boundary face method. 


\section{Introduction}

Boundary value problems involving contact are of great importance in industry related to mechanical and civil engineering, and have been the subject of research for many years. The first satisfactory analysis in theory was made by Hertz [1] in 1882 . Although many efforts $[2,3]$ were devoted to the analytical studies later, the solution is still limited to simple cases of geometry, load and boundary conditions. These limitations have motivated the development of numerical methods to solve the problem. The first numerical algorithm, known as node-to-node (NTN), imposes contact constraints between pairs of nodes. This algorithm can be found in Francavilla and Zienkiewicz [4] and Fredriksson [5] for finite element method (FEM) and in Andersson et al. [6] and París and Garrido [7] for boundary element method (BEM).

In the contact zone, the conforming discretization is required for NTN. This requirement is not an acceptable strategy of solution in some situation [8]. Thus, a non-conforming discretization method called node-to-surface approach was proposed later. In this approach, the closest point pair is applied to impose the contact constraints, so that nodes on one side of contact surfaces should be projected onto the opposite surface to obtain the information of the closest point. Nowadays the node-to-surface approach based on FEM[9-11] and BEM[12-14] has been widely used in contact modelling.

However, the node-to-surface approach presents some drawbacks both in FEM and BEM. In FEM an ad-hoc post-processing scheme is needed to recover the contact pressure, since the contact virtual work is evaluated by the contact force rather than contact pressure. And the accuracy of the resulting contact pressure is also open to question [15]. To overcome this drawback, the surface-to-surface approach (or segment-to-segment in 2D) has been proposed in [16]. The main feature of the approach is the imposition of contact constraints in a weak integral sense along the contact surface, instead of a strong, pointwise enforcement as in node-to-surface approach. Besides, the traction is included explicitly in the contact integral, so that the ad-hoc post-processing is no more necessary. Moreover, the contact patch test [17] is passed using the surface-to-surface approach. Now, in finite element the 
surface-to-surface approach has been extended to more general problem of contact and impact interactions coupled with mortar method, see e.g.[18-21]. On the other hand, a jump in contact stress [22] has been found for two dimensional contact problems in BEM when adopting the node-to-surface approach. To remedy the problem, a weak application of contact conditions [23] was proposed. In fact, the method in [23] can be interpreted as a kind of surface-to-surface approach, because the contact constraints are imposed in a weak integral manner.

So far, the surface-to-surface approach was only implemented for two-dimensional contact problems in BEM. In this paper we extend the approach to three-dimensional frictionless contact problem based on the boundary face method (BFM) [24]. The rest of this article is organized as follows: In Section 2, we introduce the discrete forms of the contact constraint definitions. Section 3 presents the boundary integral equation and the feature of BFM. Section 4 will discuss the essential difference between the node-to-surface approach and surface-to-surface approach. Numerical examples are presented to illustrate the advantages of our method in Section 5. Finally, the paper ends with conclusions in Section 6.

\section{Contact definitions}

Consider two bodies occupying domains $D^{A}, D^{B}$ and the boundary of two bodies are represented by $S^{A}$ and $S^{B}$, respectively, with reference to a fixed Cartesian coordinate system Oxyz (see Fig.1). The boundary of each body can be decomposed into two mutually disjoint sub-regions, i.e.,

$$
S^{K}=S_{C}^{K} \cup S_{N}^{K},(K=A, B)
$$

where on $S_{C}^{K}$ the two bodies come into contact, while on $S_{N}^{K}$ contact does not occur. Similarly, the $S_{N}^{K}$ of each body can be decomposed into two mutually disjoint sub-regions $S_{N U}^{K}$ and $S_{N T}^{K}$ according to the prescribed boundary conditions. Here

$$
S_{N}^{K}=S_{N U}^{K} \cup S_{N T}^{K},(K=A, B)
$$


where the displacements are known on $S_{N U}^{K}$, and the stress vectors are known on $S_{N T}^{K}$

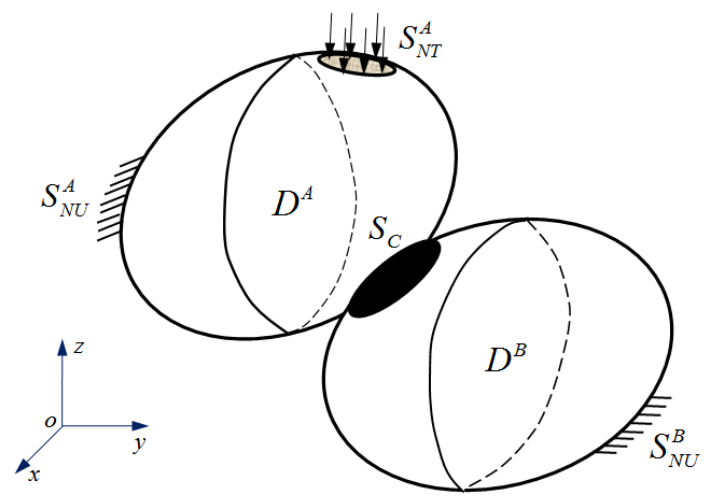

Fig.1. Problem definition

In BEM for non-conforming discretization, mutual projection should be performed between the two contact surfaces, so that the normal gap can be obtained. The typical normal gap is determined by the signed closest point distance. However, if the closest point projection is adopted for both the two contact surfaces, a asymmetry of projection [25] will occur. For the sake of simplicity, a two-dimensional contact case is illustrated in Fig.2, but the considerations below can be directly applied to the three-dimensional case.

As seen in Fig.2, the closest point for $m$ is $q$, while the closest point for $q$ is $r$. As a result, the normal gap between $m$ and $q$ is not equal to that between $q$ and $r$. Besides, when using the node-to-surface approach to enforce the contact constraints, the traction (or displacement) constraints will be imposed between $m$ and $q$ and the displacement (or traction) constraints will be imposed between $q$ and $r$. This is non-physical because the contact constraints should not be defined through three points. Thus, the closest point method is not reasonable to define the normal gap for both the two contact surfaces. To overcome the issue, we define the normal gap by closest point projection for one contact surface (from $m$ to $q$ ), while another normal gap is calculate by the distance from $q$ to the intersection $(m)$. The $m$ is determined by the intersection of a line, through $q$ and parallel to the normal vector of $q$, and the contact surface of body $\boldsymbol{A}$. 


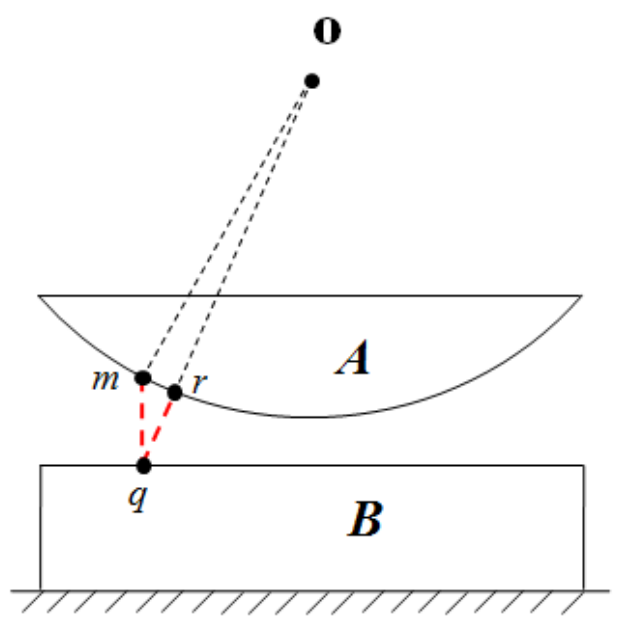

Fig.2. Closest point projection

Using this method, the contact constraints can be defined between closest point pair and constraints for nodes in the potential contact zone can be stated as following:

$$
\begin{gathered}
g_{1}=\delta_{1}-u_{1}^{A}(x)+u_{1}^{B}(x) \geq 0 \\
p_{1}=t_{1}^{A}(x)=-t_{1}^{B}(x) \leq 0 \\
p_{1} g_{1}=0
\end{gathered}
$$

where $g_{1}$ is the normal contact gap, $\delta_{1}$ is the initial normal distance between the closest point pair and $p_{1}$ is the contact pressure. The direction 1 is refer to the direction from $m$ to $q$.

\section{Boundary integral equation}

\subsection{Boundary integral formulation}

In absence of body force, the boundary integral equation can be written in the following form for each body

$$
\begin{aligned}
& C_{i j} u_{j}(P)+\int_{S} T_{i j}(P, Q) u_{j}(Q) d S(Q)=\int_{S} U_{i j}(P, Q) t_{j}(Q) d S(Q) \\
& i, j=1,2,3 P, Q \in S
\end{aligned}
$$

where $u_{j}(Q)$ and $t_{j}(Q)$ are the displacements and stress vectors of points on the boundary; $C_{i j}$ is the coefficient matrix of the free term; $T_{i j}(P, Q)$ and $U_{i j}(P, Q)$ represent the Kelvin fundamental solutions for stresses and displacements. These 
tensors, for three-dimensional case, can be expressed as following

$$
\begin{gathered}
T_{i j}(P, Q)=\frac{1}{8 \pi(1-v) r^{2}}\left[\frac{\partial r}{\partial n}\left((1-2 v) \delta_{i j}+3 r_{, i} r_{, j}\right)+(1-2 v)\left(n_{j} r_{, i}-n_{i} r_{, j}\right)\right] \\
U_{i j}(P, Q)=\frac{1}{16 \pi G(1-v) r}\left[(3-4 v) \delta_{i j}+r_{, i} r_{, j}\right]
\end{gathered}
$$

In the above equations, $G$ and $v$ are elastic constant of the body; $r$ is the distance form points $P$ to $Q$ and $n$ denotes the outward normal vector at point $Q$.

\subsection{Feature of $B F M$}

The BFM proposed by Zhang [24] is also based on the boundary integral equation. In BFM, however, the geometric data (coordinates, Jacobian and outward normal) are calculated directly from the boundary faces, which are represented in parametric form exactly as the boundary representation data structure in solid modeling. For convenience, the Fig. 3 is employed to illustrate the features.

In conventional BEM/FEM, a unique outward normal is no longer valid for linear element after discretization. For example, the outward normal at node $\boldsymbol{P}$ between the elements $S^{1}$ and $S^{2}$ is not unique as shown in Fig.3(b). So a jump in contact force will appear at node $\boldsymbol{P}$. To keep a continuous and unique outward normal at node, a weighted average of the outward normal vectors on the two adjacent elements which share the node $\boldsymbol{P}$ is defined in [18].

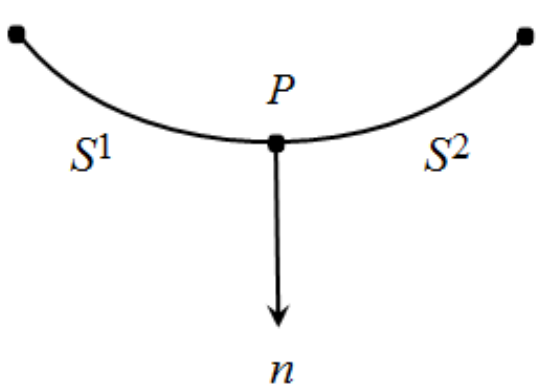

(a) BFM

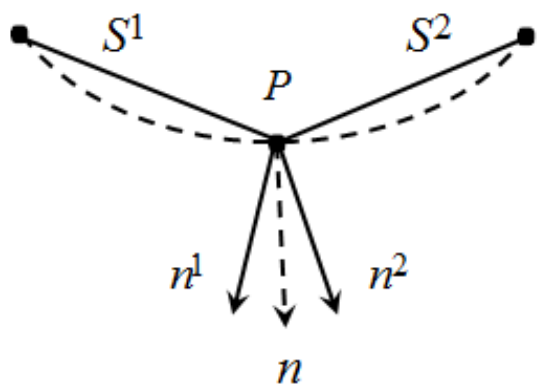

(b) BEM/FEM

Fig.3. Definition of outward normal in BFM and BEM/FEM

However, in BFM not only the uniqueness but also the continuity are valid for the 
outward normal at node. Besides, when using linear discretization the curve can be represented exactly using elements $S^{1}$ and $S^{2}$ (see Fig.3(a)), while in BEM/FEM the curve is approximated by two straight line elements as in Fig.3(b). In a word, no geometric error will be introduced in BFM, which is a truly isogeometric method [26]. Work based on the BFM can be found in references [27-30].

\section{Contact constraints method}

For any point in non-contact zone, there exist three unknown variables and three integral equations ( Eqn.(4) for $i=1,2,3$ ) can be obtained. Nevertheless, for any point in the contact zone, there exist six unknown variables and only three integral equations can be established. To make the system equation solvable, contact constraints have to be enforced. Two methods to enforce contact constraints for non-conforming discretization are discussed below.

\subsection{Node-to-surface approach}

In this method, the equilibrium is only forced at nodes of one body and the compatibility is forced at nodes of the other body. As shown in Fig.4, the traction of body $\boldsymbol{B}$ is represented by the traction shape function of body $\boldsymbol{A}$. Similarly, the displacement of body $\boldsymbol{A}$ is represented by the displacement shape function of body $\boldsymbol{B}$. In short, body $\boldsymbol{A}$ controls the tractions and body $\boldsymbol{B}$ controls the displacements. Therefore, contact constraints for the frictionless problem can be written as following:

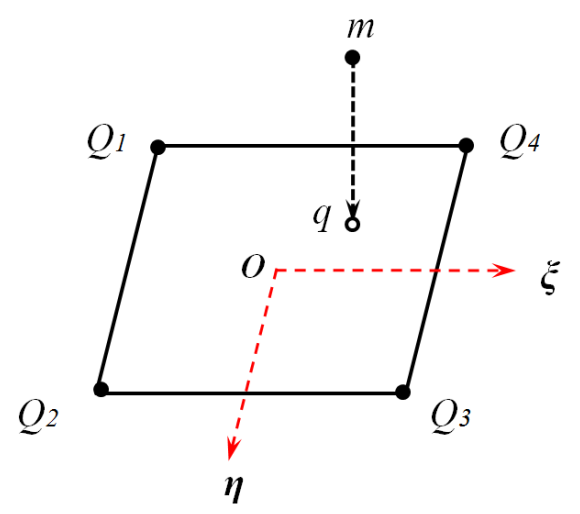

Fig.4.The node-to-surface contact model

-Equilibrium condition for nodes in the contact zone of body $\boldsymbol{B}$ 


$$
t_{1}^{B}=-\sum_{k=1}^{4} \boldsymbol{N}_{k}(\xi, \eta) t_{1}^{k, A}
$$

- Compatibility of normal displacements for nodes in the contact zone of body $\boldsymbol{A}$

$$
u_{1}^{A}=\sum_{k=1}^{4} N_{k}(\xi, \eta) u_{1}^{k, B}+\delta_{1}
$$

-Frictionless condition for nodes in contact zone

$$
t_{2}^{A}=t_{3}^{A}=t_{2}^{B}=t_{3}^{B}
$$

where $N$ is shape function. The direction 1 is the same as that in Eqn.(1) and the direction 2 and 3 are taken in any position in the plane perpendicular to the direction 1.

As we know, the forces transmitted through $S_{C}^{B}$ is equal, i.e.,

$$
\int_{S_{C}^{B}}\left(\boldsymbol{t}_{1}^{B}+\boldsymbol{t}_{1}^{A}\right) \Gamma=0
$$

and the compatibility of normal displacements in contact zone $S_{C}^{A}$ can be written as

$$
\int_{S_{C}^{A}}\left(\boldsymbol{u}_{1}^{A}-\boldsymbol{u}_{1}^{B}-\boldsymbol{\delta}_{1}\right) \Gamma=0
$$

For node-to-surface approach, the Eqn.(7) can be obtained from Eqn.(10) using collocation at nodes in $S_{C}^{B}$. Similarly, the Eqn.(8) can be obtained from Eqn.(11). Thus, the equilibrium and compatibility conditions are only forced at nodes.

\subsection{Surface-to-surface approach}

Unlike collocation method, the Galerkin method is adopted for computing the integral Eqn.(10) and (11) in this method. Then the equilibrium condition on $S_{C}^{B}$ can be written as

$$
\sum_{k}^{N C B} \int_{S_{C}^{k B}}\left(\boldsymbol{N}^{k B}\right)^{T} \boldsymbol{N}^{k B} \Gamma \boldsymbol{t}_{1}^{k B}+\sum_{k}^{N C B} \int_{S_{C}^{k B}}\left(\boldsymbol{N}^{k B}\right)^{T} \boldsymbol{N}^{A} \Gamma \boldsymbol{t}_{1}^{A}=0
$$

and the compatibility condition on $S_{C}^{A}$ can be written as

$$
\sum_{k}^{N C A} \int_{S_{C}^{k A}}\left(\boldsymbol{N}^{k A}\right)^{T} \boldsymbol{N}^{k A} \Gamma \boldsymbol{u}_{1}^{k A}-\sum_{k}^{N C A} \int_{S_{C}^{k A}}\left(\boldsymbol{N}^{k A}\right)^{T} \boldsymbol{N}^{k A} \Gamma \boldsymbol{\delta}_{1}^{k A}-\sum_{k}^{N C A} \int_{S_{C}^{k A}}\left(\boldsymbol{N}^{k A}\right)^{T} \boldsymbol{N}^{B} \Gamma \boldsymbol{u}_{1}^{B}=0
$$


where $N C B$ and $N C A$ are the number of element on contact zone $S_{C}^{B}$ and $S_{C}^{A}$ respectively, and the superscript $k$ is the index of element on which the integral is performed. Since the contact constraints are imposed in a weak integral manner, the global equilibrium and compatibility conditions can be guaranteed.

The integral of the above equations need to be calculated separately. The computation of the integrals that multiply to $\boldsymbol{t}_{1}^{k B}, \boldsymbol{u}_{1}^{k A}$ and $\boldsymbol{\delta}_{1}^{k A}$ is easy, due to the fact that only shape function and element Jacobian should be considered. However, the calculation of $\boldsymbol{G}$, the second item of Eqn.(12), depends on the overlapping area between the element $k$ of body $\boldsymbol{B}$ and element of body $\boldsymbol{A}$. Thus, a projection of element from body $\boldsymbol{B}$ onto body $\boldsymbol{A}$ is required. Meanwhile, the calculation of $\boldsymbol{M}$, the last item of Eqn.(13), also need a projection of element from body $\boldsymbol{A}$ onto body $\boldsymbol{B}$.

$$
\begin{gathered}
\boldsymbol{G}=\sum_{k}^{N C B} \int_{S_{C}^{k B}}\left(\boldsymbol{N}^{k B}\right)^{T} \boldsymbol{N}^{A} \Gamma \boldsymbol{t}_{1}^{A} \\
\boldsymbol{M}=\sum_{k}^{N C A} \int_{S_{C}^{k A}}\left(\boldsymbol{N}^{k A}\right)^{T} \boldsymbol{N}^{B} \Gamma \boldsymbol{u}_{1}^{B}
\end{gathered}
$$

If a mutually projection of the element is performed, the cost of overlapping area detection will be double. This is not a good strategy. In fact, there exists a common contact zone (overlapping area) which can be used for the integrals mentioned above. To compute the integrals, the surface-to-surface algorithm (Fig.5) will be introduced below.

\section{The surface-to-surface algorithm}

1. Project all element nodes of one contact surface (slaver) onto the boundary face (already existing in the BFM data structure) of the opposite surface (master).

2. Using a clipping algorithm [31] to find the overlapping area (common contact zone) of projected slave and master element on the boundary face.

3. Locate geometric center of polygon and divide polygon into triangular sub-elements which will be used for numerical integration. 
4. Define integration point with coordinate $\xi_{g}$ on master integration sub-elements and find the corresponding integration point $\xi_{g}^{(1)}$ on the slave element by an inverse projection.

5.Perform numerical integration for $\boldsymbol{G}$ and $\boldsymbol{M}$ on all integration sub-elements.
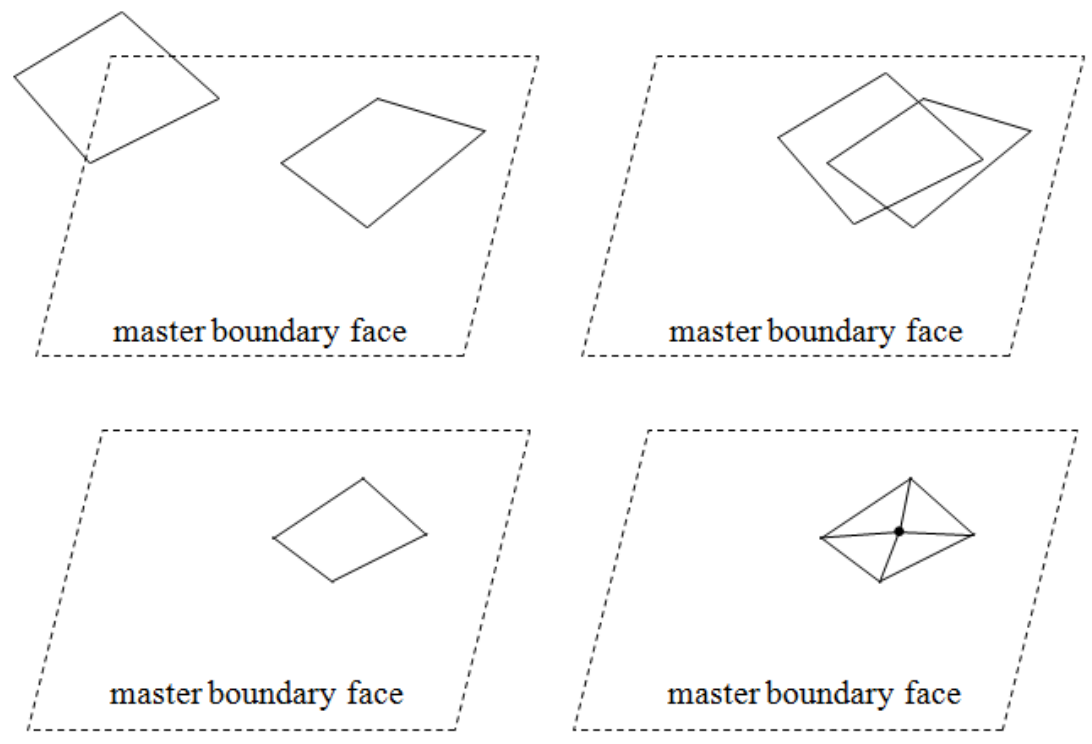

Fig.5. Main step of the surface-to-surface integration scheme

A similar integration scheme using FEM can be found in $[19,20]$. In [20] the integration scheme is called segment-based integration, and an auxiliary plane should be introduced since all element nodes need to be projected onto it to detect overlapping area. However, this is not needed for BFM because the boundary face, already existing in the BFM data structure, can replace the auxiliary plane (see [24]).

In non-conforming discretization, the contact zone $S_{C}^{A}$ is not always equal to the contact zone $S_{C}^{B}$ after discretization. Thus, the integral region of $\boldsymbol{G}$ and $\boldsymbol{M}$ should be noted. For simplicity, a two-dimensional case is shown as below

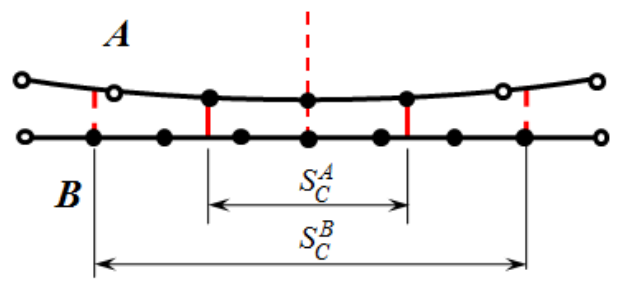

Fig.6. Contact zone of contact surfaces 
In Fig.6, the solid point denotes a contact node, while the hollow point denotes a non-contact node. Then the common contact zone $S_{C}$ is equal to $S_{C}^{A}$ which can be used for the calculation of $\boldsymbol{G}$ and $\boldsymbol{M}$. However, the $S_{C}^{B}-S_{C}^{A}$ is only applied for the calculation of $\boldsymbol{G}$. The rule is also applicable to the condition when the contact zone $S_{C}^{A}$ is larger than $S_{C}^{B}$. One way to avoid this problem is to combine the collocation method with Galerkin method, since only displacement (or traction) constraint is imposed in a weak integral manner. However, the combination is not the point of discussion in this paper.

Through the methods mentioned above, the system equation is determined. The solution procedures can be found in [32] where the node-to-surface combined with BFM has been implemented. As we know, only the boundary need to be discretized in BEM, which is also applicable to BFM. In this paper, linear quadrilateral elements are used to discretize the boundary of solids.

\section{Numerical examples}

In this section, two examples without friction are studied. The first one is used for demonstrating the feasibility of our method. The second one is presented to illustrate the advantages of our method. Meanwhile, the results of finite element software ABAQUS 6.13 are employed for comparison.

\subsection{Hertzian contact}

In Fig.7(a), a 3D Hertzian contact problem consisting of an elastic semi-cylinder ( $R=L=10$, Young's modulus $E=200$ and Poisson's ration $v=0.3$ ) and a rigid planar surface (not shown) is analyzed. A uniform pressure $p=0.1$ is applied to the top surface of the semi-cylinder. Analytical solutions for the contact pressure distribution can be characterized via the maximum normal contact pressure $p_{\max }$ and the long strip of contact width $2 a$ (see, e.g., [3]). For the given set of parameters, we obtain $p_{\max }=3.74$ and $a=0.3404$ as analytical values. The contact pressure in the cutting plane $\mathrm{x}=5$ is plotted in Fig.8. The mesh in our method is shown in Fig.7(a), having 2,396 
linear quadrilateral elements. To obtain a accurate pressure distributions, a refined mesh is used in the potential contact zone (see Fig.7 (b)). In ABAQUS, there are 298,040 linear hexahedral elements (not shown), and the mesh size in the direction of $x$ and circumferential are set to be 0.25 and 0.075 , respectively.

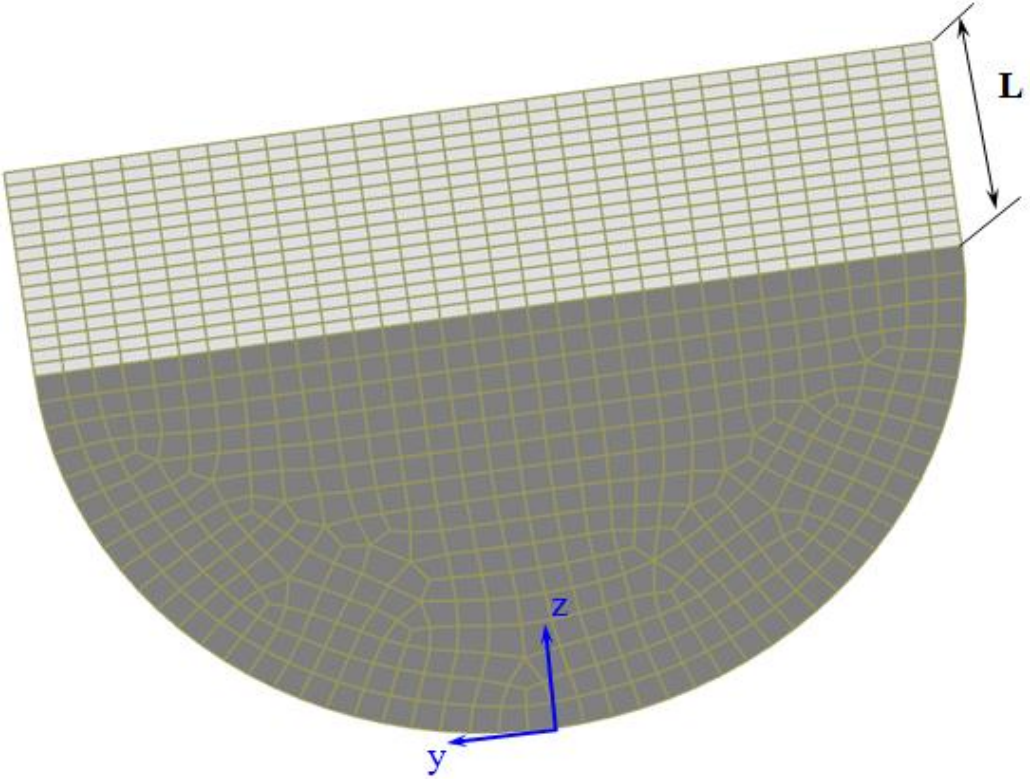

(a) geometry and mesh

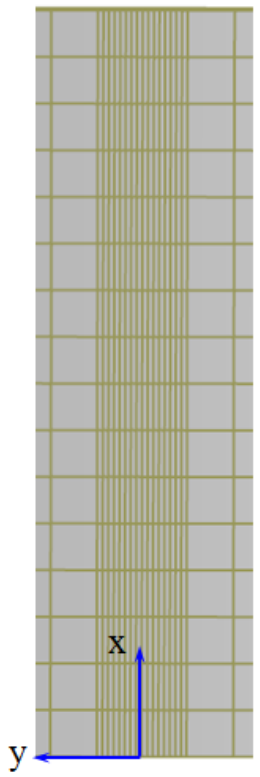

(b) mesh in contact zone

Fig.7. Mesh in our method

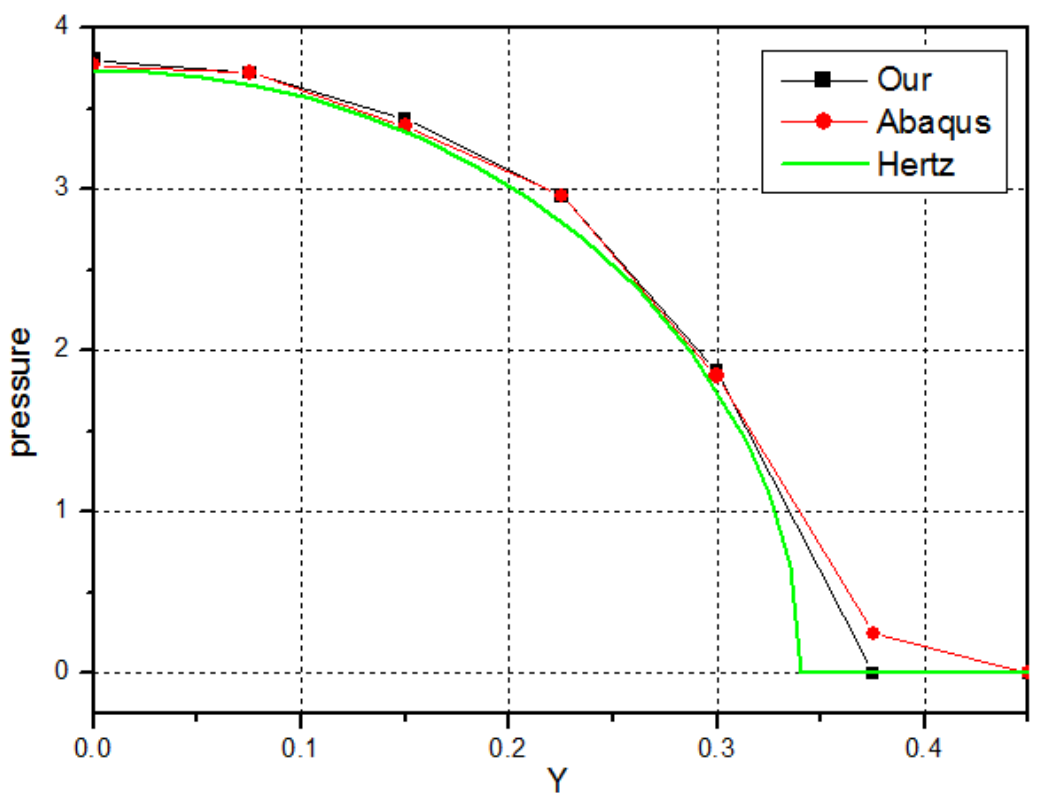

Fig.8. Contact pressure

As seen in Fig.8, the results of our method and ABAQUS are almost coincident, except in the outermost part of the contact zone. Although there is some 
discrepancy between numerical and analytical solution, the relative error of the maximum pressure in our method (3.809) with respect to the analytical solution is $1.85 \%$.

\subsection{Compression of an elastic punch on foundation}

An elastic punch is presented against a foundation as in Fig.9. The top face of the punch as well as the bottom face of foundation is a square with a length of $16 \mathrm{~mm}$. The uniform pressure $p=300 \mathrm{MPa}$ is applied on the punch's top face. Both solids are defined with equal material parameters (Young's modulus E=200GPa and Poisson's ratio $v=0.3)$.
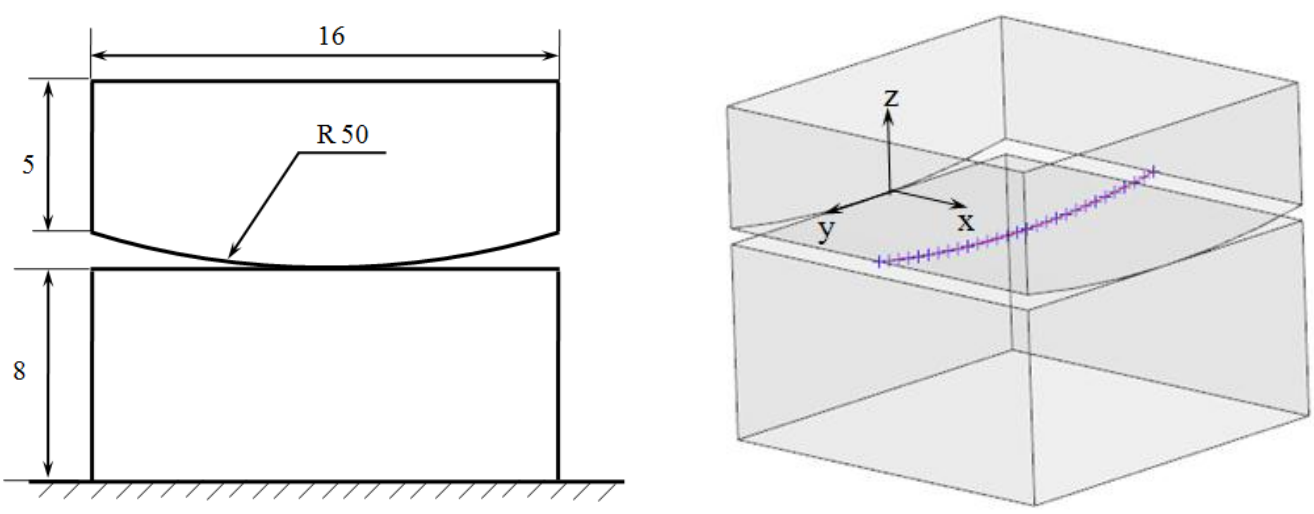

Fig.9. Elastic curve punch on foundation

In this example, the result in our method will be used to compare with that in other methods. Before comparison, a reference solution should be obtained. Thus, an ABAQUS model with three different mesh size $(0.667 \mathrm{~mm}, 0.4 \mathrm{~mm}$ and $0.32 \mathrm{~mm})$ is adopted. In ABAQUS, both the punch and foundation are modelled with linear hexahedral element. Meanwhile, the surface-to-surface discretization method and finite-sliding formulation are used. The results of red line on the punch's contact surface (see Fig.9) are employed to analyze in all later figures. 


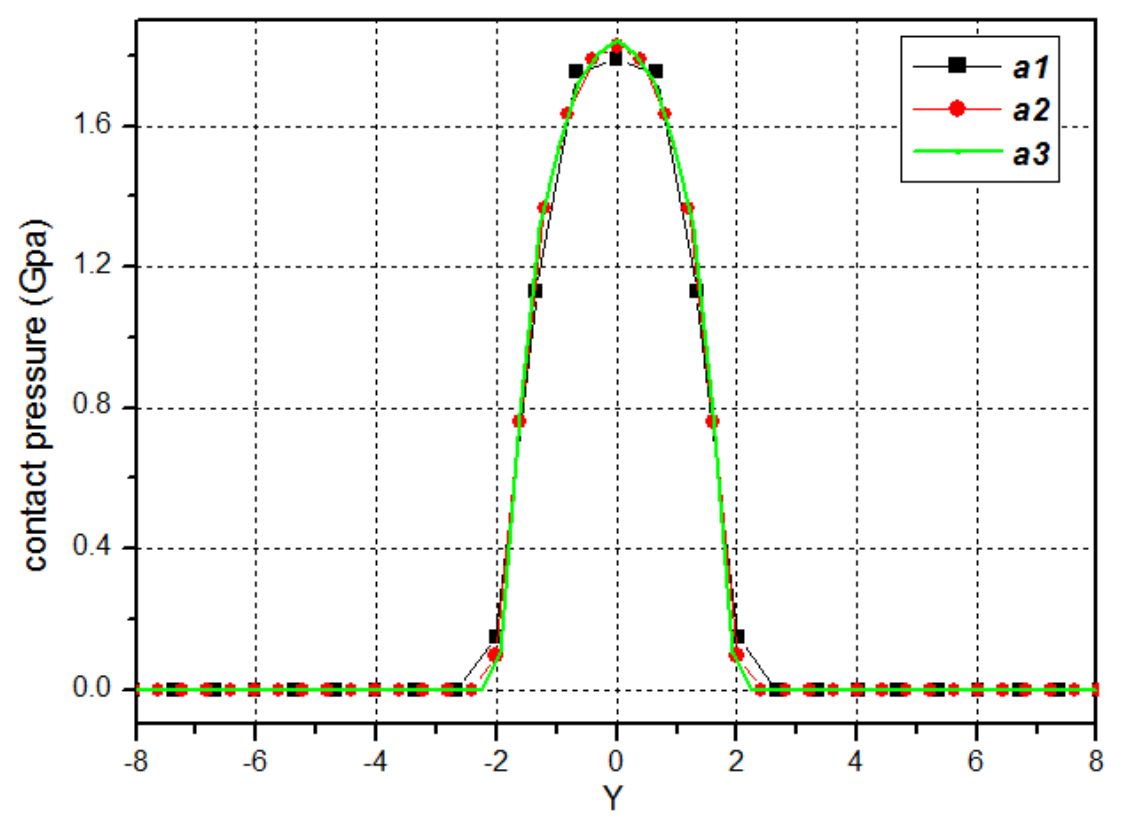

Fig.10. Contact pressure

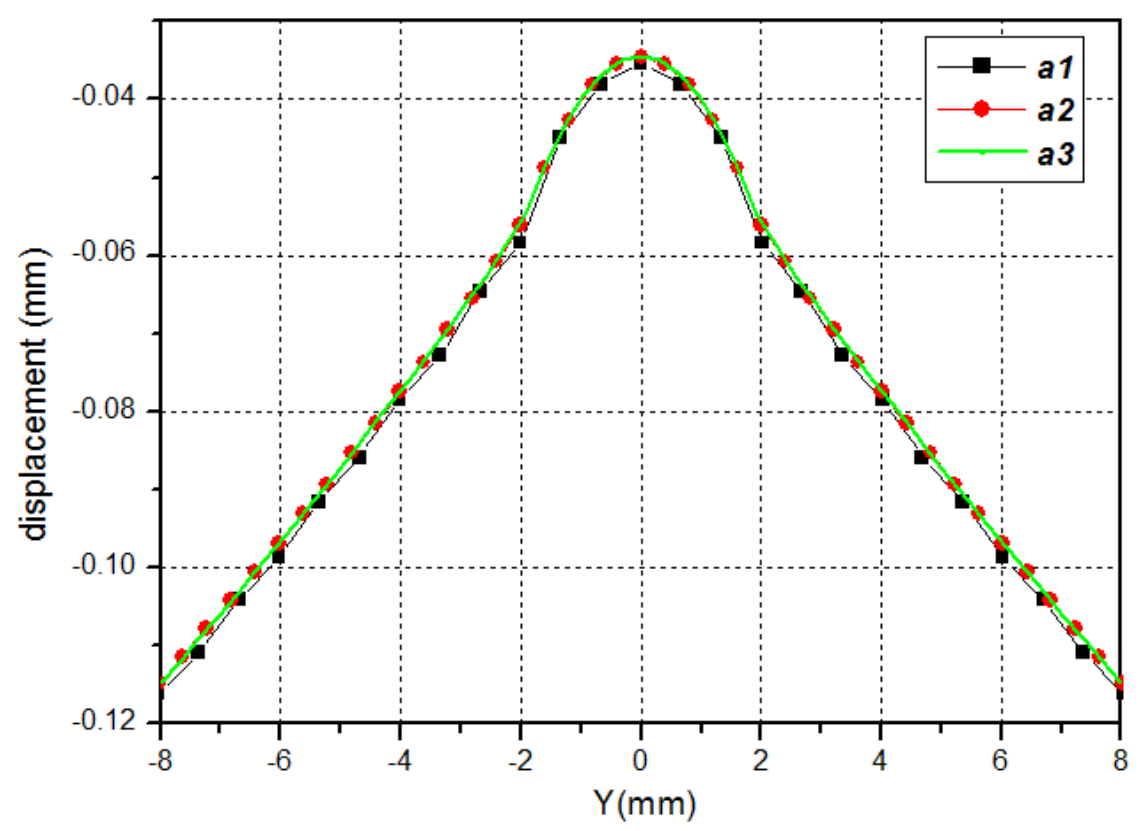

Fig.11. Displacement (Uz)

In Fig.10 and 11, the contact pressure and displacement of $\boldsymbol{a 1}(0.667 \mathrm{~mm}), \boldsymbol{a} 2$ $(0.4 \mathrm{~mm})$ and $\boldsymbol{a} 3(0.32 \mathrm{~mm})$ are exhibited. Among them, the results of $\boldsymbol{a} \boldsymbol{2}$ and $\boldsymbol{a} 3$ are coincident even having a different mesh size. That is to say, the result of $\boldsymbol{a} 3$ is stable and it can be used as a reference solution. The number of nodes and elements in $\boldsymbol{a 1}$, $\boldsymbol{a} \mathbf{2}$ and $\boldsymbol{a} \mathbf{3}$ are given in Table 1. 
Table 1. Number of nodes and elements in $a 1, a 2$ and $a 3$

\begin{tabular}{ccccc}
\hline \multirow{2}{*}{ ABAQUS } & \multicolumn{2}{c}{ Punch } & \multicolumn{2}{c}{ Foundation } \\
\cline { 2 - 5 } & Nodes & Elements & Nodes & Elements \\
\hline $\boldsymbol{a} 1$ & 5625 & 4608 & 8125 & 6912 \\
$\boldsymbol{a 2}$ & 23534 & 20800 & 35301 & 32000 \\
$\boldsymbol{a 3}$ & 44217 & 40000 & 67625 & 62500 \\
\hline
\end{tabular}

\subsubsection{Compared with ABAQUS}

As shown in Fig.12(a), the mesh size of the punch and foundation are equal and the mesh size is $0.667 \mathrm{~mm}$, so that there yield 2,150 nodes and 1,920 elements on the punch, and 2,550 nodes and 2,304 elements on the foundation in our method. This model has been solved by our method and the results are plotted in Fig.13 and 14. In the figures, the results of $\boldsymbol{a l}$ solved by ABAQUS are also plotted, since the mesh size in $\boldsymbol{a l}$ (see Fig.12(b)) is the same as that in Fig.12(a). And only the contact pressure from $-4.0 \mathrm{~mm}$ to $4.0 \mathrm{~mm}$ is plotted because the contact pressure in other part is always zero.

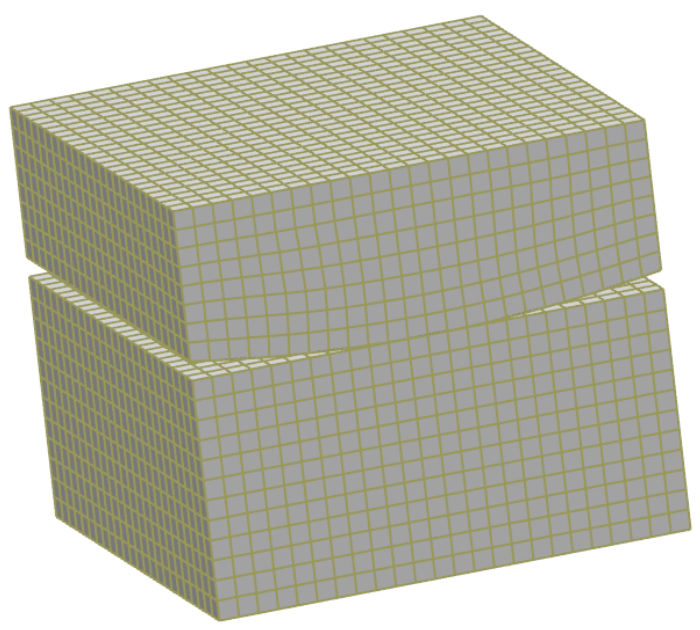

(a) Our method

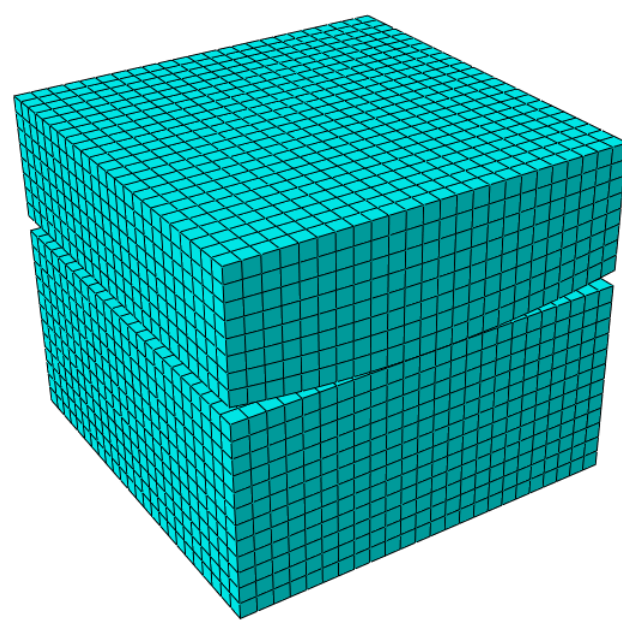

(b) $a 1$

Fig.12. Mesh 


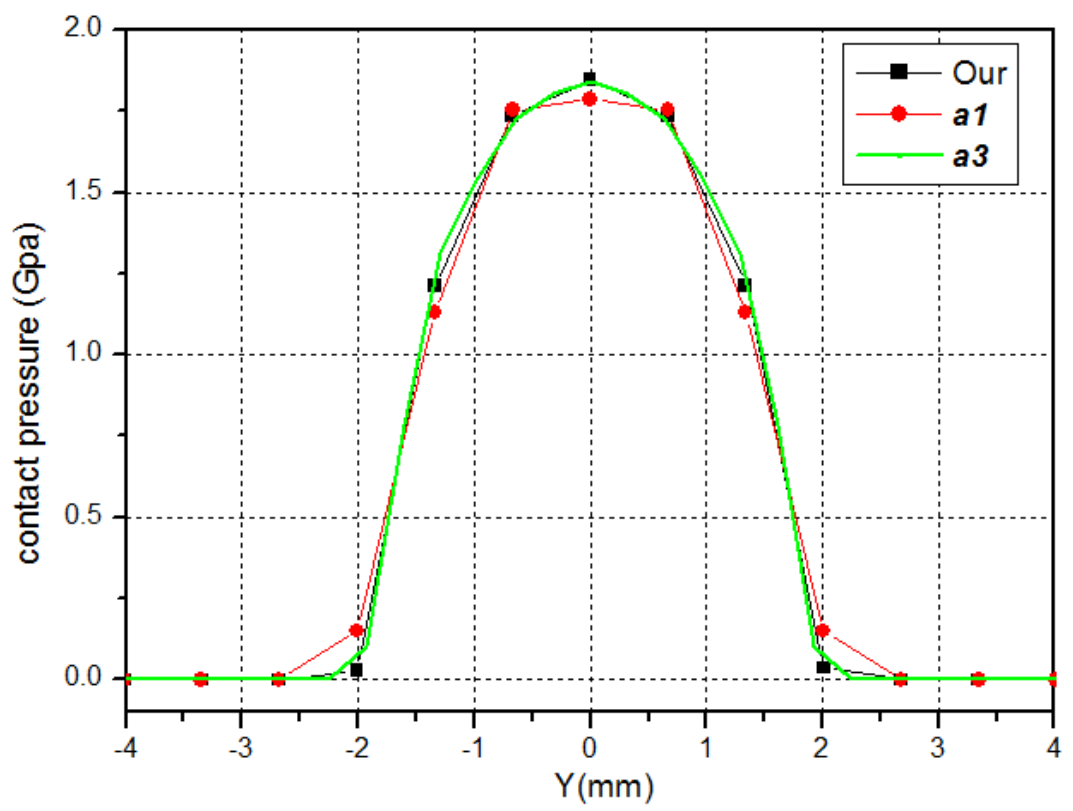

Fig.13. Contact pressure

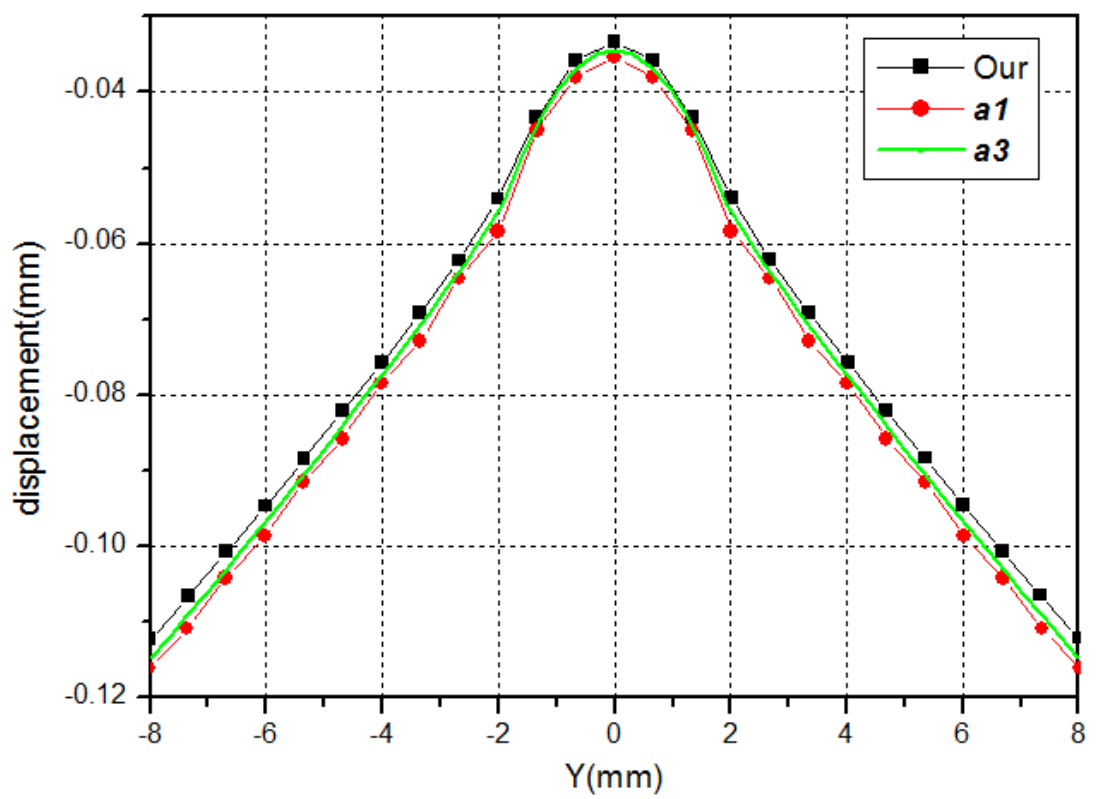

Fig.14. Displacement (Uz)

The contact pressure given in Fig.13 shows that our method has a better approximation of the reference solution a3, compared with a1. Nevertheless, the displacement exhibited in Fig.14 has some discrepancy. In the region from $-2 \mathrm{~mm}$ to $2 \mathrm{~mm}$, our method still has a good approximation of the reference solution $\boldsymbol{a} 3$, but becomes worse when far away from the center. The relative error of the displacement, at $y=8 \mathrm{~mm}$, in our method with respect to the reference solution is $2.25 \%$. The number of nodes and elements in $\boldsymbol{a} \mathbf{l}$ and $\boldsymbol{a} 3$ have been given in Table 1. 


\subsubsection{Compared with the node-to-surface approach}

In this example, the mesh sizes of the punch and foundation (see Fig.9) are $0.667 \mathrm{~mm}$ and $0.8 \mathrm{~mm}$, respectively. As a result, there yield 2,150 nodes and 1,920 elements on the punch, and 1,806 nodes and 1,600 elements on the foundation. Both node-to-surface (combined with BFM) and our method (surface-to-surface combined with BFM) are used to solve this model and the results are plotted below.

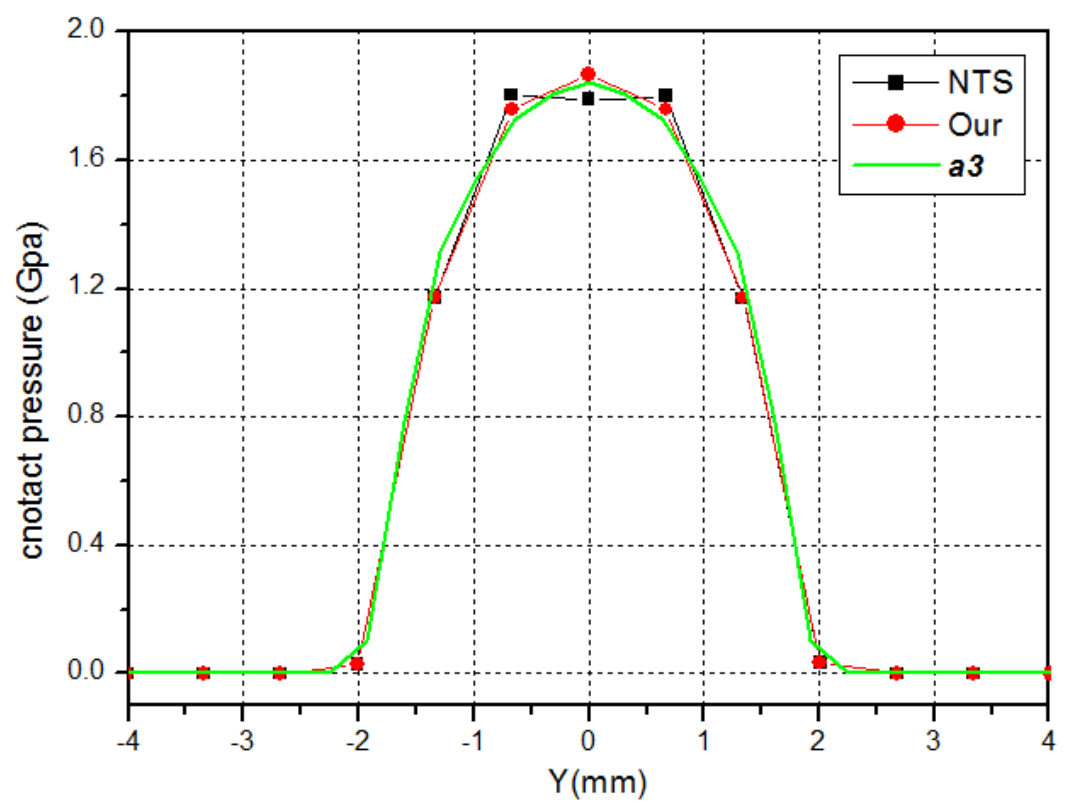

Fig.15. Contact pressure

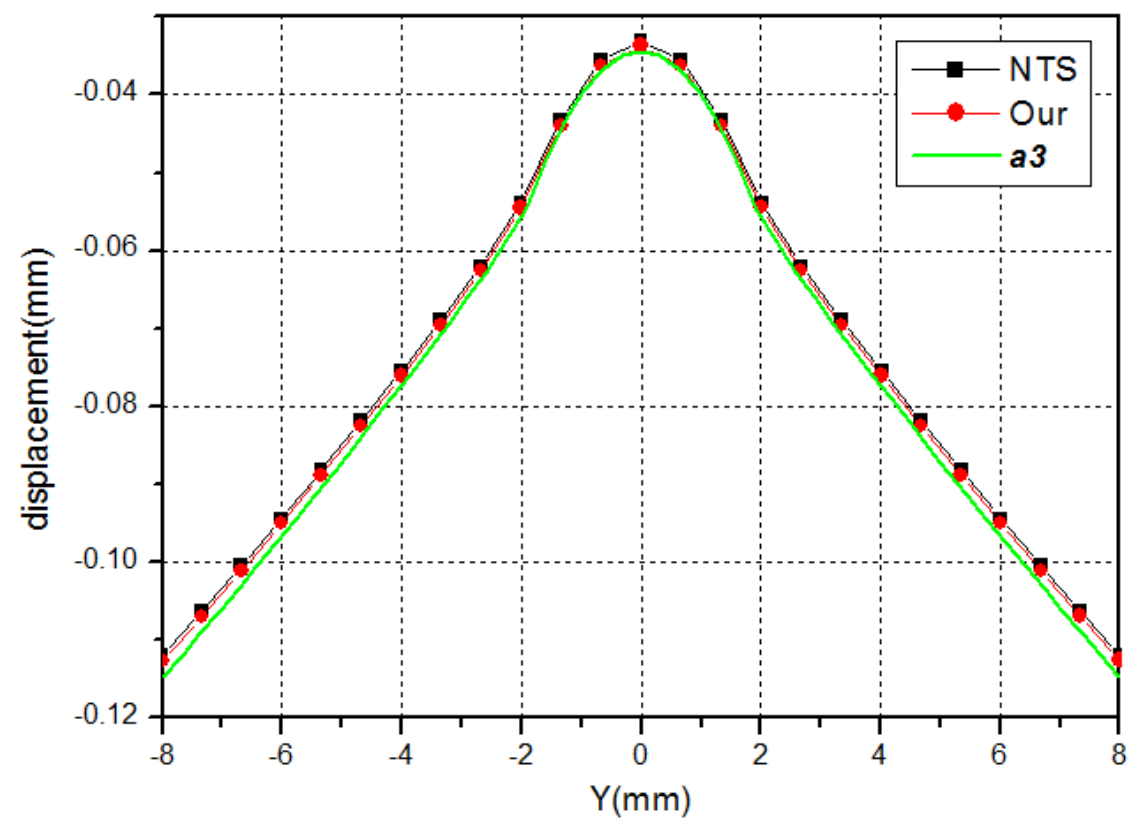

Fig.16. Displacement (Uz) 
The contact pressure results in Fig.15 illustrate that our method is more close to the reference solution $\boldsymbol{a 3}$, compared with the node-to-surface (NTS) approach. Meanwhile, the displacement results in Fig.16 show that our method is still slightly better than NTS. That is to say, our method is more accurate than NTS.

\section{Conclusions}

A surface-to-surface approach for three-dimensional frictionless contact problems has been studied by the boundary face method (BFM). The contact constraints integral equations are solved by Galerkin method rather than collocation method, so that the global equilibrium and compatibility conditions can be guaranteed. Meanwhile, the boundary face, already existing in BFM data structure, can replace the auxiliary plane, on which projection and overlapping area detection are performed. Therefore, our implementation is natural and can more precisely match the contact conditions between faces. Numerical examples show that the result of our method is encouraging and the result of surface-to-surface approach is more accurate than that of node-to-surface approach.

One disadvantage of the surface-to-surface approach is the cost of overlapping area detection for the contact constraints integral, but a more accurate solution can be obtained. To reduce the cost as much as possible, a reasonable potential contact zone is of great importance. If the potential contact zone is much larger than the final contact zone, the cost for the contact constraints integral in non-contact zone will be very expensive. Thus, a reasonable potential contact zone should be guaranteed. Our treatment is to combine node-to-surface approach with surface-to-surface approach. Firstly the node-to-surface approach is employed to solve the problem, and then a more reasonable potential contact zone can be obtained. With a more reasonable potential contact zone, the cost of surface-to-surface approach to find the final contact zone will decrease largely.

\section{Acknowledgements}

This work was supported in part by National Science Foundation of China under 
grant number 11472102, in part by Open Research Fund of Key Laboratory of High Performance Complex Manufacturing, Central South University under grant number Kfkt2013-05, in part by State Key Laboratory of Advanced Design and Manufacturing for Vehicle Body under grant number 71375003, and in part by the Collaborative Innovation Center of Major Machine Manufacturing in Liaoning.

\section{References}

[1] Hertz H. Study on the contact of elastic solids. J Reine Angew Math 1882; 92: 156-171.

[2] Gladwell GML. Contact Problems in the Classical Theory of Elasticity. Netherlands: Sijthoff and Noordhoof; 1980.

[3] Johnson KL, Contact Mechanics. Cambridge: Cambridge University Press; 1985.

[4] Francavilla A, Zienkiewicz O C. A note on numerical computation of elastic contact problems. Int J Numer Meth Eng 1975; 9(4): 913-924.

[5] Fredriksson B. Finite element solution of surface nonlinearities in structural mechanics with special emphasis to contact and fracture mechanics problems. Comput Struct 1976; 6(4): 281-290.

[6] Andersson T, Fredriksson B, Allan Persson BG. The boundary element method applied to two-dimensional contact problems. In: Brebbia CA, editor. In Proceedings of the Second International Seminar on Recent Advances in BEM. Southampton: CML Publications; 1980, p. $247-263$.

[7] Paris F, Garrido J A. On the use of discontinuous elements in two dimensional contact problems. In: Brebbia CA, editor. Boundary Elements VII. Berlin: Springer; 1985, p. 1327-1330.

[8] Blázquez A, París F. On the necessity of non-conforming algorithms for 'small displacement'contact problems and conforming discretizations by BEM. Eng Anal Bound Elem 2009; 33(2): 184-190.

[9] Bathe KJ, Chaudhary A. A solution method for planar and axisymmetric contact problems. Int J Numer Meth Eng 1985;21:65-88.

[10] Klarbring A, Bjöourkman G. Solution of large displacement contact problems with friction using Newton's method for generalized equations. Int J Numer Meth Eng 1992; 34(1): 249-269.

[11] Wriggers P. Computational contact mechanics. Berlin: Springer; 2006.

[12] Olukoko OA, Becker AA, Fenner RT. A new boundary element approach for contact problems with friction. Int J Numer Meth Eng 1993;36:2625-42.

[13] París F, Blázquez A, Canas J. Contact problems with nonconforming discretizations using boundary element method. Comput Struct 1995; 57(5): 829-839.

[14] Aliabadi M H, Brebbia C A. Computational methods in contact mechanics. Southampton Boston: Computational Mechanics Publications/Elsevier Applied Science;1993.

[15] De Lorenzis L, Wriggers P, Hughes TJR. Isogeometric Contact: A Review (ICES Report 14-09) The Institute for Computational Engineering and Sciences, The University of Texas at 
Austin, 2014.

[16] Simo J C, Wriggers P, Taylor R L. A perturbed Lagrangian formulation for the finite element solution of contact problems. Comput Methods Appl Mech Eng 1985; 50(2): 163-180.

[17] Papadopoulos P, Taylor R L. A mixed formulation for the finite element solution of contact problems. Comput Methods Appl Mech Eng 1992; 94(3): 373-389.

[18] Yang B, Laursen T A, Meng X. Two dimensional mortar contact methods for large deformation frictional sliding. Int J Numer Meth Eng 2005; 62(9): 1183-1225.

[19] Puso M A, Laursen T A. A mortar segment-to-segment contact method for large deformation solid mechanics. Comput Methods Appl Mech Eng 2004; 193(6): 601-629.

[20] Farah P, Popp A, Wall W A. Segment-based vs. element-based integration for mortar methods in computational contact mechanics. Comput Mech 2015; 55(1): 209-228.

[21] Laursen T A, Puso M A, Sanders J. Mortar contact formulations for deformable-deformable contact: past contributions and new extensions for enriched and embedded interface formulations. Comput Methods Appl Mech Eng 2012; 205: 3-15.

[22] Blazquez A, Paris F, Canas J. Interpretation of the problems found in applying contact conditions in node-to-point schemes with boundary element non-conforming discretizations, Eng Anal Bound Elem 1998; 21(4): 361-375.

[23] Blazquez A, Paris F, Mantic V. BEM solution of two dimensional contact problems by weak application of contact conditions with non-conforming discretizations. Int J Solids Struct 1998; 35:3259-3278.

[24] Zhang JM, Qin XY, Han X, Li GY. A boundary face method for potential problems in three dimensions. Int J Numer Meth Eng 2009; 80(3): 320-337.

[25] Yastrebov V A. Numerical methods in contact mechanics. London: ISTE/Wiley; 2013.

[26] Hughes TJR, Cottrell JA, Bazilevs Y. Isogeometric analysis: CAD, finite elements, NURBS, exact geometry and mesh refinement. Comput Methods Appl Mech Eng 2005; 194(39): 4135-4195.

[27] Zhou FL, Zhang JM, Sheng XM, Li GY. Shape variable radial basis function and its application in dual reciprocity boundary face method. Eng Anal Bound Elem 2011; 35(2): 244-252.

[28] Qin XY, Zhang JM, Li GY, Sheng XM. An element implementation of the boundary face method for 3D potential problems. Eng Anal Bound Elem 2010; 34(11): 934-943.

[29] Gu JL, Zhang JM, Sheng XM. B-spline approximation in boundary face method for three-dimensional linear elasticity. Eng Anal Bound Elem 2011; 35(11): 1159-1167.

[30] Zhou FL, Zhang JM, Sheng XM, Li GY. A dual reciprocity boundary face method for 3D non-homogeneous elasticity problems. Eng Anal Bound Elem 2012; 36(9): 1301-1310.

[31] J.D. Foley, A. VanDam, S.K. Feiner, J.F. Hughes. Computer Graphics: Principles and Practice(second ed.) Reading: Addison-Wesley; 1997.

[32] Zheng XS, Zhang JM, Xiong K, Shu XM. Boundary face method for 3D contact problems with non-conforming contact discretization. Eng Anal Bound Elem 2016; 63: 40-48. 